\title{
A novel technique for the measurement of the electron neutrino cross section
}

\author{
A. Longhin ${ }^{1}$, L. Ludovici ${ }^{2}$, F. Terranova ${ }^{3, a}$ \\ ${ }^{1}$ I.N.F.N., Laboratori Nazionali di Frascati, Frascati, Rome, Italy \\ ${ }^{2}$ I.N.F.N., Sezione di Roma, Rome, Italy \\ ${ }^{3}$ Department of Physics, University of Milano-Bicocca and INFN, Sezione di Milano-Bicocca, Milan, Italy
}

Received: 22 December 2014 / Accepted: 30 March 2015 / Published online: 21 April 2015

(C) The Author(s) 2015. This article is published with open access at Springerlink.com

\begin{abstract}
Absolute neutrino cross section measurements are presently limited by uncertainties on $v$ fluxes. In this paper, we propose a technique that is based on the reconstruction of large angle positrons in the decay tunnel to identify three-body semileptonic $K^{+} \rightarrow e^{+} \pi^{0} v_{e}$ decays. This tagging facility operated in positron counting mode ("event count mode") can be employed to determine the absolute $v_{e}$ flux at the neutrino detector with $\mathcal{O}(1 \%)$ precision. Facilities operated in "event by event tag mode" i.e. tagged neutrino beams that exploit the time coincidence of the positron at source and the $v_{e}$ interaction at the detector, are also discussed.
\end{abstract}

\section{Introduction}

A detailed knowledge of neutrino interaction cross sections plays a crucial role in the precision era of oscillation physics [1,2]. In the last decade, a vigorous experimental programme has been pursued, employing both the near detectors of running long-baseline experiments [3-8] and dedicated experiments [9-11] with special targets and PID capabilities. The large statistics accumulated so far and the careful strategy implemented for systematic mitigation have improved our knowledge of total and differential cross sections for $v_{\mu}$ and $\bar{v}_{\mu}$ in the range of interest $(0.3-5 \mathrm{GeV})$ for future long-baseline and sterile neutrino experiments [12]. All these experiments are, however, designed to work in $v_{e}$ appearance mode and the direct measurement of $v_{e}$ interactions still relies on scarce data $[13,14]$. Calculations are thus based on extrapolation from $v_{\mu}$ results. Despite lepton universality of weak interactions, the ratio between $v_{\mu}$ and $v_{e}$ suffers from uncertainties due to nuclear effects [15] that have to be constrained with data to reduce systematic errors

a e-mail: francesco.terranova@cern.ch in future long baseline $v_{e}$ appearance experiments $[16,17]$. To cope with this challenge, novel experimental approaches have been proposed with the aim of producing pure, intense and well controlled sources of electron neutrinos [18-22]. The technique proposed in the following has a similar aim: electron neutrinos are produced by the three body decay of $K^{+}\left(K_{e 3}\right.$, i.e. $\left.K^{+} \rightarrow e^{+} v_{e} \pi^{0}\right)$ in standard neutrino beams. The positrons are identified in the decay tunnel by purely calorimetric techniques and the beam-line is optimized to enhance the $v_{e}$ components from $K_{e 3}$ and suppress to a negligible level the $v_{e}$ contamination from muon decays. This approach - from here on called "event count mode" - has several advantages. It provides a source of electron neutrinos that can be used to study $v_{e}$ interactions in a direct manner, i.e. without relying on extrapolations from $v_{\mu}$. In addition, it delivers an observable (the positron rate) that can be directly linked to the rate of $v_{e}$ at the far detector through the three body kinematics of $K_{e 3}$. The positron rate in the decay tunnel thus determines the flux with a precision significantly better than what is currently achieved with conventional untagged $v_{\mu}$ beams $(\sim 10 \%)$. Finally, this facility paves the way for the realization of tagged neutrino beams [23-27] in the configuration proposed in Ref. [28], where the positron is associated to the corresponding $v_{e}$ interaction at the far detector on an event by event basis ("event by event tag mode"). In this mode, full kinematic reconstruction of the $K_{e 3}$ can be achieved measuring the photon pair from $\pi^{0}$ decay, thus retrieving information on the energy of $v_{e}$ for each tagged event.

The tagging concept and the rationale for the choice of the beam-line parameters, the tagging detector and the neutrino detector are introduced in Sect. 2. The beam-line up to the decay tunnel is detailed in Sect. 3 together with the expected secondary flux ( $\pi$ and $K$ ) at CERN, Fermilab, JPARC and Protvino. The decay tunnel instrumented with positron taggers and the corresponding positron identifica- 
tion performance are summarized in Sect. 4. This section also summarizes the rates and integrated doses expected at the tagger units. Background, systematics and rates at the far detector are presented in Sects. 5 and 6. Finally, perspectives for the event by event tag mode upgrade are described in Sect. 7.

\section{Conceptual design}

Unlike neutrino factories [29] and beta beams [30], conventional neutrino beams are sources of muon neutrinos from pion decays, polluted by small fractions of electron neutrinos from kaons and muons decays. The size of the contamination highly depends on the primary proton energy, on the momentum of secondaries selected by the focusing system and on the length of the decay tunnel. In general, high energy neutrino beams as the CNGS [31] are contaminated by $v_{e}$ originating from the $K_{e 3}$ decays of $K^{+}$while the contamination of lower energy neutrino beams is mostly due to $\pi^{+} \rightarrow \mu^{+} v_{\mu} \rightarrow e^{+} v_{e} \bar{v}_{\mu} v_{\mu}$. The $v_{e}$ flux depends on the hadron production yield on the target and on the acceptance of the focusing and transport system to the decay tunnel. Even with dedicated hadro-production data, pion monitoring at the target and muon monitoring at the beam dump, the uncertainty on the size of this contamination has never been reduced below $10 \%$. It is a fair educated guess that for a conventional facility, a dedicated effort, including ancillary experiments to measure the kaon production rate in replica targets, might reduce this uncertainty to a level not lower than $7-8 \%$.

The ratio between the $v_{e}$ from $K_{e 3}$ and the $v_{\mu}$ from pion decay can be enhanced increasing the energy of the selected secondaries and reducing the length of the decay tunnel (Fig. 1 - black lines). This comes at the expenses of the overall neutrino flux. The $v_{e}$ beam contamination from muon decays in flight (DIF) is also reduced (Fig. 1 - red lines). The $v_{e} / v_{\mu}$ ratio scales as

$R_{K / \pi} \cdot B R\left(K_{e 3}\right) \cdot \frac{\left[1-e^{-L / \gamma_{K} c \tau_{K}}\right]}{\left[1-e^{-L / \gamma_{\pi} c \tau_{\pi}}\right]}$

where $R_{K / \pi}$ is the ratio between $K^{+}$and $\pi^{+}$produced at the target and transported down to the entrance of the decay tunnel. $B R\left(K_{e 3}\right)$ is the $K_{e 3}$ branching ratio: $5.07 \pm 0.04 \%$ [32]. $L$ is the length of the decay tunnel. $\tau_{K}\left(\tau_{\pi}\right)$ and $\gamma_{K}\left(\gamma_{\pi}\right)$ are the lifetime and Lorentz factor of the $K^{+}\left(\pi^{+}\right)$, respectively. The scaling of Eq. 1 is depicted in Fig. 1 assuming $R_{K / \pi}=10 \%$ (see Table 1 below).

For a beam dominated by the $K_{e 3}$ contamination, the only source of primary positrons in the decay tunnel is the $K^{+} \rightarrow \pi^{0} e^{+} \nu_{e}$ decay and the Dalitz from the $\pi^{0} \rightarrow e^{+} e^{-} \gamma$ decay $(\mathrm{BR} \simeq 1.2 \%)$. All other positrons are either due to DIF of muons (from pions or beam halo) or to photon

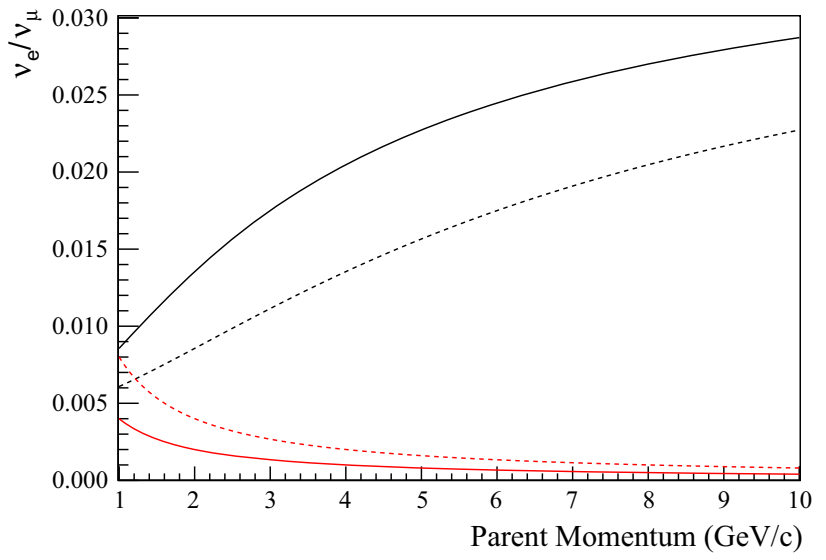

Fig. 1 Black lines approximate scaling (see Eq. 1) of the $v_{e} / v_{\mu}$ fluxes as a function of the momentum of secondaries. The continuous (dashed) line corresponds to a $50 \mathrm{~m}(100 \mathrm{~m})$ decay tunnel. The red lines show the approximate scaling of the $v_{e} / v_{\mu}$ from muon DIF

conversions in the material around the decay tunnel. Two body positron decays $\left(\pi^{+} \rightarrow e^{+} v_{e}\right.$ and $K^{+} \rightarrow e^{+} v_{e}$ ) are chirality-suppressed and can be neglected. As a consequence, all primary positrons are originated by three-body decays and are distributed at angles much larger than the angles of the muons from two-body $\pi^{+} \rightarrow \mu^{+} v_{\mu}$ decays. For the beam parameters considered in Sect. 3, the mean positron angle $(88 \mathrm{mrad})$ is 22 times larger than the corresponding mean $\mu^{+}$angle and $\sim 30$ times larger than the beam divergence of the undecayed particles. These considerations [28] support the instrumentation of the decay tunnel with detectors having a geometry similar to the calorimeters of hadron colliders (hollow cylinders). As discussed in Sect. 4, the technology requirements (radiation hardness, fast readout, fast recovery time for pile-up mitigation etc.) are quite similar, too. Since neither the muons from $\pi^{+}$decay nor the bulk of undecayed particles cross the calorimeter before reaching the beam dump, the particle rate is much smaller than the rate of muon monitors in conventional neutrino beams; such rate (see below) can be handled by standard detector and readout technologies developed for the hadron colliders.

Finally, the need for short decay tunnels reduces the size of the calorimeter and makes the instrumentation of the whole tunnel - which was considered far-fetched in 1979 [24] - a viable option.

\section{Production and transport of secondaries}

The proposed facility is based on a conventional beam-line with primary protons impinging on a target, producing secondary hadrons which are captured, sign selected and transported further down to the instrumented decay tunnel (see Fig. 2; Sect. 4). Inclusive secondary pion yields on solid targets increase linearly with proton energy but the tech- 
Fig. 2 Layout of the facility (not to scale)

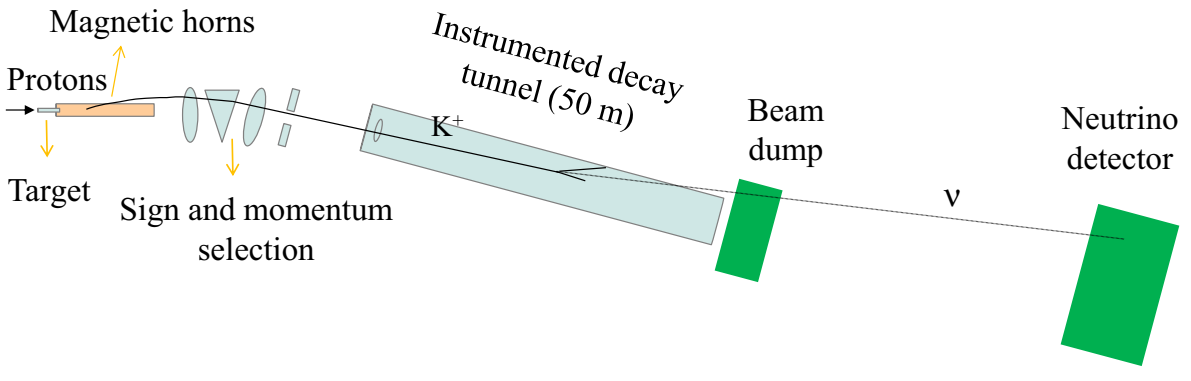

nique presented in this study exploits high energy kaons to enhance the $\pi^{+} / e^{+}$separation at the calorimeters (Sect. 5) and to reduce the decay losses after the focusing system. The optimal value for the mean secondary momentum is around $8.5 \mathrm{GeV}$. Lower values decrease the $\pi^{+} / e^{+}$separation efficiencies, while higher values reduce the flux and bring the $v_{e}$ spectrum above the region of interest for future long-baseline experiments $(0.5-4 \mathrm{GeV})$.

In the following we assume to collect secondary positive particles $\left(\pi^{+}, K^{+}\right)$produced at the target and to transport them to the entrance of the decay tunnel with a momentum bite of $\pm 20 \%$ centered at $8.5 \mathrm{GeV}$. The decay tunnel consists of an evacuated beampipe ( $40 \mathrm{~cm}$ radius) surrounded by the positron detectors (see Sect. 4). For the calculation of the neutrino flux, we simulated pions and kaons distributed uniformly in a $10 \times 10 \mathrm{~cm}^{2}$ window in the transverse plane and with a flat polar angle distribution (up to $3 \mathrm{mrad}$ ). In fact, the actual meson beam distribution at the entrance of the decay tunnel is not a critical parameter because the neutrino beam divergence at this energy is dominated by the large neutrino decay angle with respect to the parent meson. The meson beam emittance has only to be small enough to contain the secondary beam inside the tagging detector. An unfocused meson beam entering the decay tunnel within a window of $\pm 5 \mathrm{~cm}$ in both transverse projections and with a polar angle smaller than $3 \mathrm{mrad}$, is fully contained in a $50 \mathrm{~m}$ long, $40 \mathrm{~cm}$ radius decay tunnel, even including the tertiary muons produced in $\pi^{+}$decays (see Sect. 4). This phase space area corresponds to a geometrical acceptance of the decay tunnel $A=4 \times(5 \mathrm{~cm}) \times(3 \mathrm{mrad})=4 \epsilon_{x x^{\prime}}=4 \epsilon_{y y^{\prime}}=0.60 \mathrm{~mm} \mathrm{rad}$, where $\epsilon_{x x^{\prime}}=\epsilon_{y y^{\prime}}=0.15 \mathrm{~mm}$ rad in both transverse projections. Here $x, y$ and $x^{\prime}=\mathrm{d} x / \mathrm{d} z, y^{\prime}=\mathrm{d} y / \mathrm{d} z$ are respectively the particle positions and slopes transverse to the direction $z$ of the beam.

As discussed in Sect. 7, a long extraction ( $>10 \mathrm{~ms})$ is needed only for the event by event tag operation mode. The capture of secondaries at the target in a facility operated in event count mode can thus be implemented with conventional magnetic horns. On the other hand, fast extractions $(10 \mu \mathrm{s})$ challenge the positron tagger, whose local rate must be kept at the level of $\mathcal{O}(1) \mathrm{MHz} / \mathrm{cm}^{2}$. The optimal choice for the event count mode is $\sim 2 \mathrm{~ms}$. Such extraction length has already been employed at the CERN West Area Neutrino
Facility (WANF [33]). It is also the parameter on which the NUMI horns and their power supplies have been originally designed [34]. Actually, in spite of the fact that both NOVA and $\mathrm{T} 2 \mathrm{~K}$ implement a fast extraction (10 $\mu \mathrm{s})$ scheme [35], the typical current pulse width used to source their horns is $\sim 2$ ms. Longer extractions introduce additional constraints on the horn, due to the increase of Joule heating. In particular, thicker conductors will be needed to reduce resistive heating which, in turn, can cause beam deterioration due to particle re-interactions [34]. In addition, long extractions may reduce the $v_{e}$ CC purity due to cosmic background at shallow depth (see Sect. 6).

Downstream of the horn, the secondary beam is transported to the decay tunnel entrance by a transfer line based on quadrupolar magnets for the focusing and bending dipoles for the momentum selection. Along the transfer line, at the bending section, the high energy residual primary protons are separated and transported to a dump [21].

In order to evaluate the secondary meson yields in this study we have used Fluka $2011[36,37]$ to simulate primary proton interactions on a $110 \mathrm{~cm}$ long (about 2.6 interaction lengths) cylindrical beryllium target of $3 \mathrm{~mm}$ diameter. For the momentum bite considered, the secondary yields at the target highly depend on the primary proton energy. We considered here proton energies of 30, 50, 60, 70, 120 and $450 \mathrm{GeV}$. These correspond to facilities based on the JPARC proton synchrotron $(30 \mathrm{GeV})$, the upgrade of the U-70 accelerator in Protvino (50-70 GeV) [38], the primary proton beamline of NUSTORM (60 GeV) [21], the Main Injector at Fermilab (120 GeV) used for the NUMI beam [39], the CERN-SPS operated in low energy mode [40] (120 GeV) and the full energy CERN-SPS [41] (450 GeV).

The capture and transfer line has not been simulated in this work as it requires a site-dependent dedicated study that is beyond the scope of the paper. To evaluate the fluxes at the entrance of the decay tunnel we used the phase space $x x^{\prime}, y y^{\prime}$ of pions and kaons in a momentum bite of $8.5 \mathrm{GeV} / \mathrm{c} \pm 20 \%$ at $5 \mathrm{~cm}$ downstream the $110 \mathrm{~cm}$ long target. We assume that all secondaries within an emittance $\epsilon_{x x^{\prime}}=\epsilon_{y y^{\prime}}=0.15 \mathrm{~mm}$ rad are focused with a typical horn focusing efficiency of $85 \%$ (see e.g. Tables VIII-X of Ref. [21]). These particles are captured and transported down to the entrance of the decay tunnel. The ellipse of this area best 
Table 1 Pion and kaon yields for horn focusing at $(8.5 \pm 1.7) \mathrm{GeV} / \mathrm{c}$. The rightmost column is computed assuming a 500 ton neutrino detector

\begin{tabular}{lllll}
\hline$E_{p}(\mathrm{GeV})$ & $\begin{array}{l}\pi^{+} / \mathrm{PoT} \\
\left(10^{-3}\right)\end{array}$ & $\begin{array}{l}K^{+} / \mathrm{PoT} \\
\left(10^{-3}\right)\end{array}$ & $\begin{array}{l}\text { PoT for a } 10^{10} \\
\pi^{+} \text {spill }\left(10^{12}\right)\end{array}$ & $\begin{array}{l}\text { PoT for } 10^{4} \\
v_{e} \text { CC }\left(10^{20}\right)\end{array}$ \\
\hline 30 & 4.0 & 0.39 & 2.5 & 5.0 \\
50 & 9.0 & 0.84 & 1.1 & 2.4 \\
60 & 10.6 & 0.97 & 0.94 & 2.0 \\
70 & 12.0 & 1.10 & 0.83 & 1.76 \\
120 & 16.6 & 1.69 & 0.60 & 1.16 \\
450 & 33.5 & 3.73 & 0.30 & 0.52 \\
\hline
\end{tabular}

matching the phase space distribution downstream the target, i.e. the one maximising the pion flux, is selected and the mesons lying within the ellipses in both transverse planes are summed up.

The results are summarized in Table 1 . The second and third columns show the pions and kaons per proton on target (PoT) transported at the entrance of the decay tunnel. The fourth column shows the number of PoT in a single extraction spill to obtain $10^{10}$ pions per spill. The last column shows the number of integrated proton on target that are needed to reach $10^{4} v_{e} \mathrm{CC}$ events on a 500 tons neutrino detector (see Sect. 6), i.e. to enable a measurement of the $v_{e}$ absolute cross section with a statistical precision of $1 \%$ (larger statistics may be needed to measure differential cross sections with a single bin precision of $\mathcal{O}(1 \%)$. Positron counting, however, is mandatory only for the absolute cross section normalization). These proton fluxes are well within the reach of the above-mentioned accelerators both in terms of integrated PoT (from $5 \times 10^{20}$ at $30 \mathrm{GeV}$ to $5 \times 10^{19}$ at $450 \mathrm{GeV})$ and protons per spill $\left(2.5 \times 10^{12}-3 \times 10^{11}\right)$. With respect to present running modes, two changes have to be envisaged. The machine must provide proton pulses with ms duration as in the former CERN-WANF (as already mentioned, current neutrino beams are operated with pulse durations of $\sim 10 \mu \mathrm{s})$. In addition, since the integrated number of spills is large $\left(\sim 2 \times 10^{8}\right.$ for proton pulses producing $10^{10}$ $\pi^{+}$per spill - see third column of Table 1), the accelerator should be run either with a repetition rate of several Hertz or in multi-turn extraction mode in order to have enough proton bursts well separated in time hitting the target.

A notable exception is the U-70 synchrotron, which cannot be used in its present form since the average power is less than $10 \mathrm{~kW}$ at $60 \mathrm{GeV}$ and the data taking (see Table 1) would exceed 6 years. The performance of a U-70 based facility will depend on the final outcome of the OMEGA Project [38]. An average power in $\mathrm{U}-70$ of $100 \mathrm{~kW}$ at $70 \mathrm{GeV}$ would imply a $\sim 1$ year long data taking assuming an effective yearly run of 200 days at nominal power. All other accelerators considered in Table 1 can be employed without additional upgrades. Low energy drivers are, however, slightly favored due to the higher repetition rate already available.
Table 2 Pion and kaon yields forward $(80 \mu \mathrm{Sr})$ at $(8.5 \pm 1.7) \mathrm{GeV} / \mathrm{c}$. The rightmost column is computed assuming a 500 ton neutrino detector

\begin{tabular}{lllll}
\hline$E_{p}(\mathrm{GeV})$ & $\begin{array}{l}\pi^{+} / \mathrm{PoT} \\
\left(10^{-3}\right)\end{array}$ & $\begin{array}{l}K^{+} / \mathrm{PoT} \\
\left(10^{-3}\right)\end{array}$ & $\begin{array}{l}\text { PoT for a } 10^{10} \\
\pi^{+} \text {spill }\left(10^{12}\right)\end{array}$ & $\begin{array}{l}\text { PoT for } 10^{4} v_{e} \\
\mathrm{CC}\left(10^{20}\right)\end{array}$ \\
\hline 30 & 0.24 & 0.027 & 42 & 72 \\
50 & 0.58 & 0.069 & 17 & 28 \\
60 & 0.73 & 0.091 & 14 & 22 \\
70 & 0.80 & 0.095 & 13 & 20 \\
120 & 1.25 & 0.16 & 8.0 & 12.2 \\
450 & 3.65 & 0.43 & 2.7 & 4.6 \\
\hline
\end{tabular}

The magnetic horns cannot be pulsed for times much longer than $10 \mathrm{~ms}$, such as the long extraction needed to operate the tagged beam facility in event by event mode. An alternative to the horns for the mesons capture is the use of purely static focusing and transport systems based on large aperture quadrupoles/dipoles [53]. In all these schemes, however, the capture is limited to the very forward secondaries produced at target. As a reference, Table 2 shows the pion and kaon yields within the momentum bite, $(8.5 \pm 1.7) \mathrm{GeV} / \mathrm{c}$ and a forward $80 \mu \mathrm{Sr}$ acceptance [28]. Clearly, the large gain in flux due to the horn-based focusing system compared with static systems simplifies remarkably the design and construction of the event count mode facility.

Since this angular acceptance is small, a Lithium lens could be possibly used for the focusing of secondaries downstream the target. However, operation of Li-lenses with the $\mathcal{O}(1)$ s extraction times needed for the event by event tag mode has still to be demonstrated.

Compared to the yields of Tables 1 and 2, we expect a reduction of the kaon yield due to decay in the transport line $(\sim 16 \%$ for an overall length of $10 \mathrm{~m})$ and finite capture and transport efficiency. Similarly, the use of graphite or INCONEL [21] targets will increase the secondary yield by $10-40 \%$. The yields of Table 1 thus represent an approximation of particle production and transport down to the instrumented decay tunnel. The precision is, however, appropriate for the aim of this study.

\section{The instrumented decay tunnel}

The decay tunnel is a $50 \mathrm{~m}$ long evacuated beam pipe, surrounded by a calorimeter that consists of a hollow cylinder with $R_{\text {in }}=40 \mathrm{~cm}$ inner radius and $R_{\text {out }}=57 \mathrm{~cm}$ outer radius (see Fig. 3). The inner radius corresponds to a line of sight between the entrance of the tunnel and the beam dump of 8 mrad. All undecayed particles $\left(\pi^{+}, K^{+}, p\right)$ and all muons from the 2-body decay of $\pi^{+}$will reach the dump without crossing the calorimeter. The overall rate at the calorimeter will therefore be dominated by kaon decays. Since the decay 


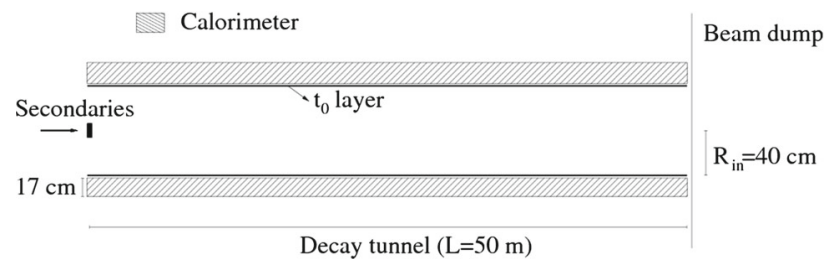

Fig. 3 The instrumented decay tunnel (not to scale). The black rectangle on the left indicates the entrance window of the secondary particles in the transverse plane $( \pm 5 \mathrm{~cm})$

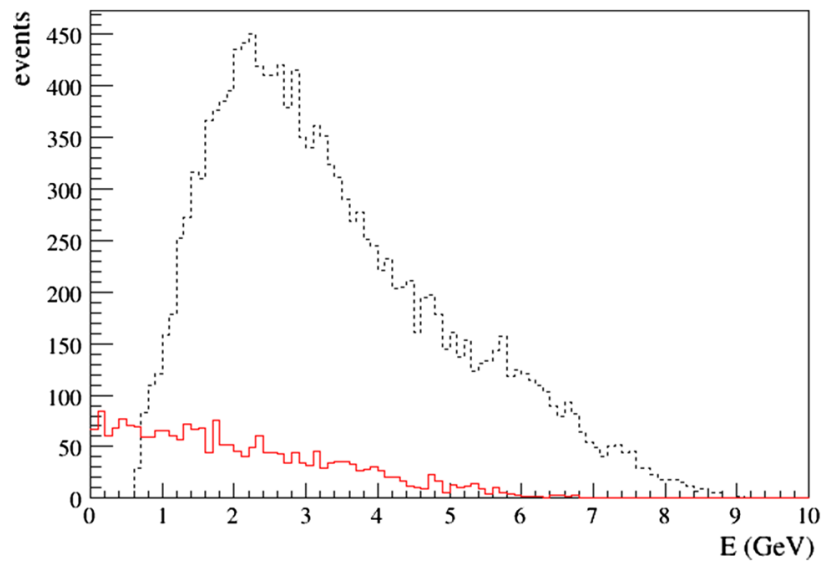

Fig. 4 Energy distribution of positron (red continuous line) and pions (black dashed) from kaon decays hitting the calorimeter for $10^{5} \mathrm{~K}^{+}$at the entrance of the decay tunnel

products are forward going, the calorimeter is thick enough to provide containment for nearly all particles originating from kaon decays. The energy and angle distribution of the positrons from $K_{e 3}^{+}$decays is shown in Figs. 4 and 5 (red continuous line). The mean polar angle of the positrons is $88 \mathrm{mrad}$. Figure 4 (black dashed line) also shows the energy distribution of background $\pi^{+}$from 2-body decay of $K^{+}$ (see Sect. 5). Positrons in the decay tunnel are identified by calorimetric techniques, exploiting the longitudinal shower development for particle identification. Photon rejection is achieved by a " $t_{0}$ layer", a pre-shower that provides the absolute time of arrival of the charged particle and is used to veto neutral particles in the calorimeter.

Figure 6 shows the number of particles (expressed in $\mathrm{Hz} / \mathrm{cm}^{2}$ ) entering the calorimeter as a function of the position $z$ along the tunnel. Each bin corresponds to a surface of $2 \pi R_{i n} \Delta z=1.26 \mathrm{~m}^{2}$. For a $2 \mathrm{~ms}$ extraction length and $10^{10}$ $\pi^{+}$per spill, the maximum positron rate (upper plot - red dashed line) is $10 \mathrm{kHz} / \mathrm{cm}^{2}$. The overall rate (upper plot black continuous line) is dominated by muons originating by two-body decays of kaons $\left(K_{\mu 2} \equiv K^{+} \rightarrow \mu^{+} v_{\mu}\right.$, whose BR is $63.55 \pm 0.11 \%$; see lower plot - black continuous line) and photons (lower plot - green dotted line). The peak rate is $500 \mathrm{kHz} / \mathrm{cm}^{2}$ ( $5 \mathrm{MHz}$ per channel for a calorimeter with a granularity of $10 \mathrm{~cm}^{2}$ ). Due to the $3 \mathrm{mrad}$ beam divergence and the Lorentz boost of decayed particles, rates are low in

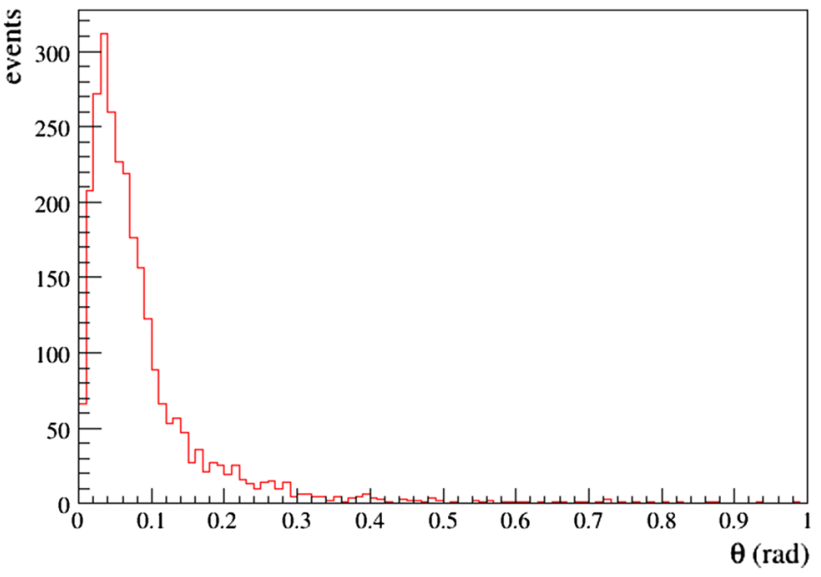

Fig. 5 Polar angle distribution of positrons for $10^{5} K^{+}$at the entrance of the decay tunnel. Small angle positrons, i.e. $e^{+}$reaching the beam dump without crossing the calorimeter are included

the first $10 \mathrm{~m}$ of the tunnel and they saturate at nearly constant value for $z>10 \mathrm{~m}$. During a single $2 \mathrm{~ms}$ spill, we expect $10^{10} \pi^{+}$and $1.02 \times 10^{9} \mathrm{~K}^{+}$at the entrance of the tunnel (the $K^{+} / \pi^{+}$ratio is $10.2 \%$ for $120 \mathrm{GeV}$ protons - see Table 1$)$. The number of kaon decays per spill is $5.6 \times 10^{8}$ (1 decay each $4 \mathrm{ps}$ ) and the corresponding number of positrons from $K_{e 3}$ is $2.8 \times 10^{7}$.

The particle decays in the tunnel, the crossing of the $t_{0}$ layer and the calorimeter response to charged and neutral particles have been simulated through GEANT4 $[42,43]$. In this study the calorimeter is simulated as a homogenous copper cylinder (radiation length $X_{0}=1.44 \mathrm{~cm}$, nuclear interaction length $\lambda_{I}=15.3 \mathrm{~cm}$ ). Choices other than copper as the absorber material (e.g. steel or lead-steel hybrid systems) are also worth consideration in terms of cost-effectiveness, ease of machining and nuclear properties.

In case of full longitudinal containment (electrons and pions), the reconstructed energy $E_{t o t}$ in the calorimeter is based on the true particle energy smeared according to the following parametrization:

$\frac{\sigma_{E}}{E}=\frac{95 \%}{\sqrt{E(\mathrm{GeV})}} \oplus 7 \%$ for hadrons

$\frac{\sigma_{E}}{E}=\frac{13 \%}{\sqrt{E(\mathrm{GeV})}} \oplus 3 \%$ for $e^{-}, e^{+}, \gamma$

which correspond to typical performance of sampling calorimeters. Note that the low-density active material is not simulated. Hence, the actual outer radius of the calorimeter will be larger than $R_{\text {out }}$ (by $\sim 30 \%$ for $1.5 \mathrm{~cm} \mathrm{Cu}$ slabs interleaved by $0.5 \mathrm{~cm}$ scintillator tiles).

The simulated energy deposition is sampled at the first $5 X_{0}\left(E_{1}\right)$ and $10 X_{0}\left(E_{2}\right)$ and the variables $R_{1} \equiv E_{1} / E_{\text {tot }}$ and $R_{2} \equiv E_{2} / E_{\text {tot }}$ are used for pion/positron separation. Since uncertainties on $E_{1}$ and $E_{2}$ are completely dominated by fluctuations due to lateral leakage, $E_{1}\left(E_{2}\right)$ is defined as 
Fig. 6 Upper plot The black continuous (red dashed) line shows the overall particle (positron) rates in the calorimeter as a function of the $z$ position along the instrumented tunnel. Lower plot Muon (black continuous), photon (green dotted) and pion (blue dot-dashed line) rates in the calorimeter as a function of the $z$ position. Rates are computed for a $2 \mathrm{~ms}$ extraction length and $10^{10} \pi^{+}$per spill
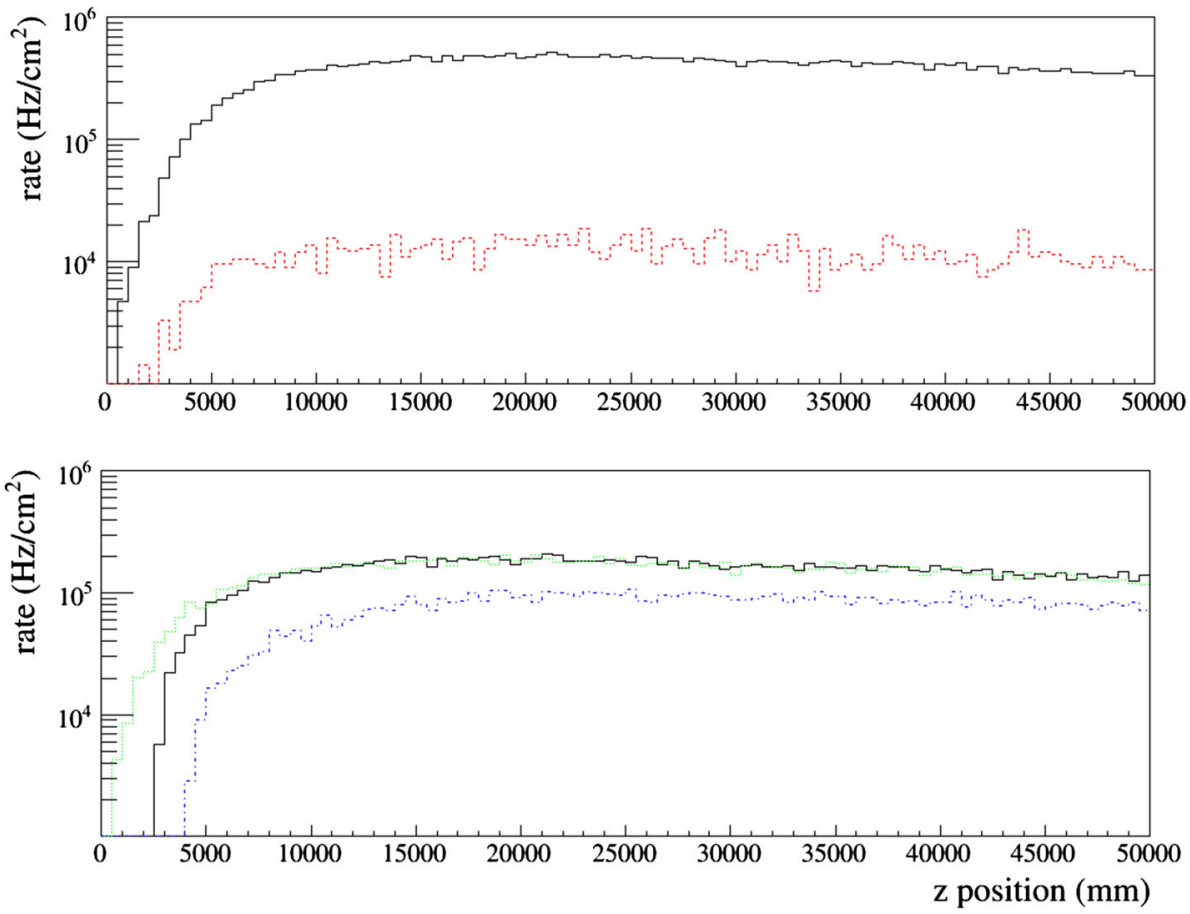

Table 3 Positron efficiency after cuts

\begin{tabular}{ll}
\hline Cut & Efficiency $(\%)$ \\
\hline$K_{e 3}$ decay & 100 \\
$e^{+}$in calorimeter & 85 \\
$R_{1}, R_{2}$ cuts & 67 \\
$E_{\text {tot }}>300 \mathrm{MeV}$ & 59 \\
\hline
\end{tabular}

the energy deposited in the first 5 (10) radiation lengths inside a cylinder of radius $2 R_{M}$ without additional smearing. $R_{M}=$ $1.568 \mathrm{~cm}$ is the Moliere radius of copper. The material of the $t_{0}$ layer and beam pipe is neglected: the impact of the beam pipe material on the background from photon conversion is discussed in Sect. 5.

A positron is defined as an energy deposit in the calorimeter associated with a hit in the $t_{0}$ layer. The energy deposited $E_{\text {tot }}$ must be greater than $300 \mathrm{MeV}$. We also request the energy deposit in the first 5 and $10 X_{0}$ to be significantly larger than for a minimum ionizing particle (MIP): $R_{1}>0.2$, $R_{2}>0.7$. These requirements select positrons from $K_{e 3}$ with $69 \%$ efficiency. Table 3 summarizes the overall efficiency (59\%) including the geometrical acceptance of the tagging calorimeter due to positrons escaping at low polar angles into the beam dump. Since local rates result only from kaons and are quite low compared with collider requirements, several technologies are available both for the calorimeter and for the $t_{0}$ layer. As a reference, we considered a scintillator tile calorimeter readout by SiPM and WLS fibers similar to the CALICE AHCAL [44] but with much coarser longitudinal segmentation. Other options, developed both for LHC and for
CLIC are possible, too [45]. Unlike applications at colliders, pile-up mitigation and integrated doses are not particularly critical. For a $R=0.5 \mathrm{MHz} / \mathrm{cm}^{2}$ local rate and a tile size $S \simeq 10 \mathrm{~cm}^{2}$, the pile up probability is

$P=R S \Delta T_{\text {cal }}$

$\Delta T_{\text {cal }}$ being the recovery time of the calorimeter. It corresponds to $P=0.05$ for $\Delta T_{\text {cal }}=10 \mathrm{~ns}$. In fact, pile-up mostly results from the overlap of a muon from $K_{\mu 2}$ with a candidate positron. Further pile-up mitigation is possible since MIP-like deposits and punch-through particles can be vetoed or removed offline using the longitudinal segmentation of the calorimeter and, if needed, a muon catcher layer (Fe + muon chambers) located at $R>R_{\text {out }}$.

Similarly, the integrated dose is not a critical parameter for this facility. From Table 1, $10^{4} v_{e} \mathrm{CC}$ events are obtained at the neutrino detector from the DIF of $1.94 \times 10^{17}$ kaons. The deposited energy of decayed kaons is 150 MJ. Most of this energy ( $64 \%$, i.e. the $K_{\mu 2} \mathrm{BR}$ ) is either uniformly distributed in the copper volume (muons) or lost outside the tunnel (neutrinos). Assuming conservatively that all residual energy is deposited in the first $3 X_{0}$ of the calorimeter and that the calorimeter extends from $z=10$ to $z=50 \mathrm{~m}$, the corresponding integrated dose is $<1260$ Gray.

In event count mode, several technologies are available for the $t_{0}$ layer since the detector operates mainly as a photon tagger. For an event by event tag facility (see Sect. 7), however, the $t_{0}$ layer must match or exceed the time resolution of the neutrino detector. Plastic scintillator tiles offer $<1$ ns resolution [46] with $\mathcal{O}(10) \mathrm{cm}^{2}$ granularities. Con- 
ventional silicon detectors are not appropriate because large surfaces increase the detector capacitance and deteriorate the time resolution. Low gain avalanche detectors [47] can overtake this limitation making a semiconductor based $t_{0}$ layer a viable option.

In general, the technology choice will mostly be driven by cost effectiveness. If the decay tunnel is instrumented from $z=10$ to $z=50 \mathrm{~m}$, the corresponding calorimeter mass is 185 tons and the surface of the $t_{0}$ layer is $100.5 \mathrm{~m}^{2}$. For a $10 \mathrm{~cm}^{2}$ granularity and three longitudinal samples in the calorimeter, the overall number of channels is thus $\sim 4 \times 10^{5}$.

\section{Background}

The $K_{e 3}$ branching ratio represents only $\sim 5 \%$ of the overall kaon decays. The bulk of particles crossing the calorimeter is due to muons from the $K_{\mu 2}$ decay mode and pions from the fully hadronic mode $\left(K^{+} \rightarrow \pi^{+} \pi^{0}\right.$ with $B R=(20.66 \pm$ $0.08) \%$ [32]). The calorimetric muon/positron separation is excellent since minimum ionizing particles (muons and punch-through pions) cluster at low values of $R_{1}$ and $R_{2}$. The misidentification rate is below $10^{-3}$ when integrated to all muons produced by $K_{\mu 2}, K_{\mu 3}$ and the DIF of pions from the other decay modes. As a consequence, background from muon misidentification does not represent a limitation for PID in the instrumented decay tunnel.

$\pi^{+} / e^{+}$separation is much less efficient and dominates the positron background. The main contribution comes from the two-body fully hadronic decay mode $K^{+} \rightarrow \pi^{+} \pi^{0}$. The spectrum of charged pions from this decay mode is shown in Fig. 4 together with the positron signal. Again, pions cluster at lower values of $R_{1,2}$ but charge exchange and the intrinsic fluctuation of the e.m. component in hadronic showers can mimic a positron, especially at low energy. The integrated misidentification probability is $\epsilon_{\pi^{+} \rightarrow e^{+}}=2.2 \%$ and the contamination in the positron signal due to pion misidentification amounts to $13 \%$. Possible overlaps between the photon from $\pi^{0}$ decay and the pions are included in the simulation and, as well as Dalitz $\pi^{0}$ decays, give negligible contributions. Pile-up has not been included since its effect is marginal (see Sect. 4).

Additional contributions to background come from the $K^{+} \rightarrow \pi^{+} \pi^{+} \pi^{-}$decay mode (BR $\simeq 5.6 \%$ ). In spite of the smaller BR, the higher charge multiplicity results into a larger misidentification efficiency and increases the overall background contamination to $18 \%$. Unlike $K^{+} \rightarrow \pi^{+} \pi^{0}$, the $K^{+} \rightarrow \pi^{+} \pi^{+} \pi^{-}$three-prong decays can be vetoed requiring no identified charged pions pointing to the decay vertex. This background reduction technique, however, needs to exploit the granularity of the detectors to identify the decay vertex along $z$ and the time resolution of the $t_{0}$ layer. For the case under study ( $2 \mathrm{~ms}$ extraction and $10 \mathrm{~cm}^{2}$ granularity), the precision on $z$ is $\sim 1 \mathrm{~m}$ and the average time among $K^{+}$ decays into charged pions in a $1 \mathrm{~m}$ section of the tunnel is 510 ps. As a consequence, multi-prong background reduction sets the scale of the $t_{0}$ layer time resolution to $\mathcal{O}(100) \mathrm{ps}$. In the present analysis, this reduction technique has not been considered.

Photons produced in the decay tunnel originate from $\pi^{0}$ decays in the semileptonic and hadronic modes of the kaons. The most important source is $K^{+} \rightarrow \pi^{+} \pi^{0}$. These photons will not give a hit in the $t_{0}$ layer associated with the energy deposit in the calorimeter. Photons converted in the material inside the $t_{0}$ layer can constitute a background because the event count mode facility does not exploit time correlation among particles ( $\pi^{+}$and $\gamma$ 's in this case) or the other particles can lay outside the geometrical acceptance of the calorimeter. For a $1.5 \mathrm{~mm}$ Be beam-pipe [48], the conversion rate is $3 \times 10^{-3}$ and, even without additional background mitigation techniques, the contamination is less than $2 \%$. It grows to $6 \%$ for a $1 \mathrm{~mm} \mathrm{Al}$ vacuum tanks. Again, if the $t_{0}$ layer has a time resolution of $\mathcal{O}(100) \mathrm{ps}$, this background can be suppressed to a negligible level vetoing prompt pions that originate from the same area of the candidate positron. The photon background is also negligible if the $t_{0}$ layer is installed inside the vacuum pipe, as for the Large Angle Veto calorimeters of NA62 [49]. All sources of background are summarized in Table 4 together with the $\epsilon_{\pi^{+} \rightarrow e^{+}}$misidentification probability.

\section{Rates at the neutrino detector and systematic errors}

The beamline and instrumented tunnel of Sects. 3 and 4 produce a neutrino beam that is enriched in $v_{e}$ from kaon decays and depleted in $v_{e}$ from muon DIF. Assuming a far detector located $100 \mathrm{~m}$ from the entrance of the tunnel $(50 \mathrm{~m}$ from the beam dump), 500 ton mass (isoscalar target) and a crosssectional area ${ }^{1}$ of $17.7 \times 17.7 \mathrm{~m}^{2}, 2.1 \times 10^{-3} v_{\mu} /$ PoT cross the detector for $120 \mathrm{GeV}$ protons. The number of electron neutrinos from $K^{+}$decays is $3.8 \times 10^{-5} v_{e} / \mathrm{PoT}$ and the $v_{e}$ from DIF is $1.2 \times 10^{-6} v_{e} / \mathrm{PoT}$. Since the $K^{+} / \pi^{+}$ratio is nearly constant from 30 to $450 \mathrm{GeV}$ (see Table 1 ), the $v_{e} / v_{\mu}$ flux ratio at the neutrino detector is independent of the proton energy and is:

$$
\begin{aligned}
& \frac{\Phi_{v_{e}}}{\Phi_{v_{\mu}}}=1.8 \%\left(v_{e} \text { from } K_{e 3}\right) \\
& \frac{\Phi_{v_{e}}}{\Phi_{v_{\mu}}}=0.06 \%\left(v_{e} \text { from DIF }\right)
\end{aligned}
$$

As expected, the beam is enriched in $v_{e}$ from kaon decays, while the contamination of $v_{e}$ from DIF is negligible. The

\footnotetext{
${ }^{1}$ It corresponds to the surface of a cylindrical detector with a geometrical acceptance of $100 \mathrm{mrad}$.
} 
Table 4 Sources of background and misidentification probability

\begin{tabular}{lllll}
\hline Source & BR & Misid & $\epsilon_{X \rightarrow e^{+}}(\%)$ & Contamination \\
\hline$\pi^{+} \rightarrow \mu^{+} v_{\mu}$ & $100 \%$ & $\mu \rightarrow e$ misid. & $<0.1$ & Neglig. (outside acceptance) \\
$\mu^{+} \rightarrow e^{+} \bar{v}_{\mu} v_{\mu}$ & DIF & genuine $e^{+}$ & $<0.1$ & Neglig. (outside acceptance) \\
$K^{+} \rightarrow \mu^{+} v_{\mu}$ & $63.5 \%$ & $\mu \rightarrow e$ misid. & $<0.1$ & Negligible \\
$K^{+} \rightarrow \pi^{+} \pi^{0}$ & $20.7 \%$ & $\pi \rightarrow e$ misid. & 2.2 & $13 \%$ \\
$K^{+} \rightarrow \pi^{+} \pi^{+} \pi^{-}$ & $5.6 \%$ & $\pi \rightarrow e$ misid. & 3.8 & $5 \%$ \\
$K^{+} \rightarrow \pi^{0} \mu^{+} v_{\mu}$ & $3.3 \%$ & $\mu \rightarrow e$ misid. & $<0.1$ & Negligible \\
$K^{+} \rightarrow \pi^{+} \pi^{0} \pi^{0}$ & $1.7 \%$ & $\pi \rightarrow e$ misid. & 0.5 & Negligible \\
\hline
\end{tabular}

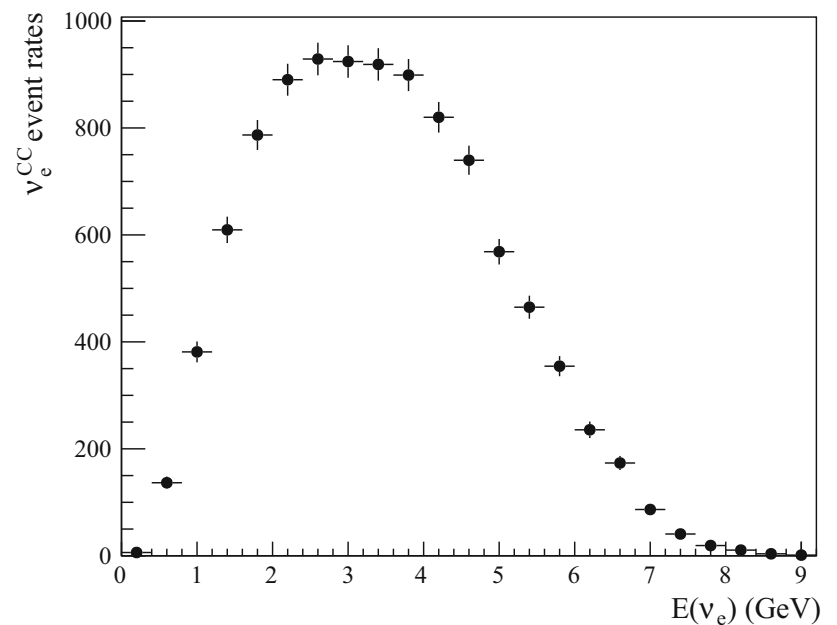

Fig. 7 Energy distribution of the $v_{e} \mathrm{CC}$ events

positrons at the calorimeter are therefore proportional to the number of electron neutrino crossing the detector. The small difference from the naïve scaling of Fig. 1 is mostly due to $v_{\mu}$ from $K^{+}$decays (not included in Eq. 1).

The event rate has been estimated folding the incoming flux with the corresponding $v_{e} \mathrm{CC}$ cross-section. The spectrum of $v_{e} \mathrm{CC}$ events at the detector for $E_{v}>0.3 \mathrm{GeV}$ is shown in Fig. 7 (the events in the first bin correspond to $0.3<E_{v}<0.4 \mathrm{GeV}$ ). The mean energy is $3 \mathrm{GeV}$ with a FWHM of $\sim 3.5 \mathrm{GeV}$.

The number of positrons reconstructed in the calorimeter is directly proportional to the flux of $v_{e}$ at the source. This provides a direct measurement of the $v_{e}$ flux, independent of the hadron production yield, the $K / \pi$ ratio, the secondary transport efficiency and the number of integrated PoT, i.e. of the main source of flux systematic errors in cross section measurements. It depends, however, on the geometrical efficiency of the neutrino detector, the knowledge of the positron efficiency in the calorimeter and on the background. In fact, only $\sim 80 \%$ of the tagged positrons will produce neutrinos that cross the detector and $\sim 15 \%$ of the $v_{e}$ CC observed at the detector will remain untagged since the corresponding positron is lost in the beam dump. In turn, this implies that the geometrical efficiency will also depend on the kinematics of $K_{e 3}$ decay and on the actual divergence of the beam at the entrance of the tunnel. Finally, the geometrical efficiency slightly depends on the slope of the hadron energy distribution in the momentum bite.

All beam parameters describing the spatial distribution of kaons at the entrance of the tunnel can be measured monitoring the charged pions in dedicated low-intensity proton extractions with negligible statistical uncertainty. As for standard collider applications, the PID separation capability of the calorimeter will be measured in test-beams before the installation and can be cross-checked on site. Although a detailed assessment of systematics requires a full simulation of the beamline and the detector response, to best of current knowledge the overall systematic budget can be kept within $\mathcal{O}(1 \%)$.

If the facility is operated in event count mode, the time resolution constraint on the neutrino detector are loose and the technology choice is mostly driven by the neutrino detection efficiency and the corresponding systematics [50]. For electron identification, scintillator based detectors offer fast time response $(<10 \mathrm{~ns})$ and good energy resolution but the granularity and PID capability is limited by the size of the scintillator cells.

Liquid Argon (LAr) detectors have superior granularity and PID capabilities, thus achieving a smaller systematic error associated to $\pi^{0}$ mis-identification background. On the other hand, the longer integration time in LAr detectors results in pile-up of signal events and cosmic background. In particular the proton extraction length $(2 \mathrm{~ms})$ matches the integration time of LAr detectors. Unlike fast extraction beams, the timing of the event with the proton current profile will be less effective for cosmic rejection even if a scintillator based fast trigger [51] is used. According to [52], LAr detectors equipped with an active veto system can be operated even with moderate overburden and sub-GeV electron neutrinos.

Finally, it is worth mentioning that a facility operated in event count mode can be run with reversed polarity to measure the $\bar{v}_{e}$ cross section. In addition, the high energy $v_{\mu}$ CC subsample is mostly due to the $K_{\mu 2}$ decays; it can thus be employed in combination with the positron rate (or, more directly, with the tagged large-angle muon rate) to retrieve information on the $v_{\mu} \mathrm{CC}$ cross section. These applications have not been considered in the present study. 


\section{Event by event tag}

The beam parameters of Sect. 3 cannot be used to run the facility in event by event tag mode. For a $2 \mathrm{~ms}$ proton extraction, one decay every 4 ps will be observed on average at the tagging detector. The average time difference between positrons would be $\sim 70 \mathrm{ps}$, which corresponds to a time resolution at the limit both of current technologies and of the intrinsic limitation of this method (see below).

In the event by event tagging facility, the time coincidence is performed between the timing of the neutrino interaction and the timing of the positron. Since the neutrino production vertex is unknown, the timing difference is corrected for the time of flight between the neutrino interaction vertex and the position of the positron tag in the decay tunnel. A neutrino is uniquely associated to a positron, i.e. it is flavor tagged as an electron neutrino on an event by event basis, if the time difference $\delta t$ between the tagging detector and the neutrino detector is compatible with $\Delta / c$ within the timing uncertainties. Here, $\Delta$ is the distance between the neutrino interaction vertex and the point along the decay tunnel axis at the same longitudinal position as the positron impact point on the tagging detector. Since both the neutrino and positron emission angles are small, this is a good approximation of the neutrino position at the time of the positron tagging. For a tagging calorimeter of inner radius $R_{i n}$, the average correction to $\Delta$ due to the positron emission angle would be of the order of $O\left(R_{i n} \theta / 2 c\right)$ ps, or $\sim 80$ ps for an average positron angle of $\theta=88 \mathrm{mrad}$. The intrinsic limit of the time coincidence can be conservatively assumed of the same order, due to the spread of the positron emission angle distribution. The uncertainty in the time coincidence due to detector resolution of the neutrino vertex and positron tagging positions can be estimated in $O(50)$ ps.

The requirements on the timing resolution can be loosened well above the intrinsic limit increasing the proton extraction length. A $1 \mathrm{~s}$ extraction would bring the average time between two decays to $1.5 \mathrm{~ns}$ and the average time among $K_{e 3}$ decays to $30 \mathrm{~ns}$. An event by event tag facility could hence be designed employing existing technologies. Defining $\delta$ as the linear sum of the $t_{0}$-layer and neutrino detector time resolution, the accidental tag probability $\mathcal{A}$ is

$$
\begin{aligned}
\mathcal{A} \equiv & {\left[N _ { K } \cdot \operatorname { B R } ( K _ { e 3 } ) \left(1-e^{\left.\left.-\frac{\gamma_{K} c \tau_{K}}{L}\right) \epsilon+\mathrm{bkg}\right]}\right.\right.} \\
& \cdot \delta \simeq 2 \times 10^{7} \frac{\delta}{T_{\text {extr }}}
\end{aligned}
$$

where $N_{K}$ the number of kaons per second, $L$ the length of the decay tunnel, $\epsilon$ the overall tagging efficiency, "bkg" the background contamination and $T_{\text {extr }}$ the proton extraction length. Eq. 5 sets the scale for the overall time resolution that is needed to build a tagged neutrino beam with rates at the detector similar to the event count facility discussed above. $\mathcal{A}$ is $2 \%$ for $\delta \simeq 1 \mathrm{~ns}$.

An event by event tag facility offers several advantages, including the possibility to veto the intrinsic contamination of conventional beams for every observed event at the far detector [27] and to measure the neutrino energy reconstructing the kinematics of $K_{e 3}$. It can also be used to measure the $v_{\mu}$ cross section from the $K^{+} \rightarrow \mu^{+} v_{\mu}$ decays, tagging the large angle muons in the decay tunnel and counting the $v_{\mu} \mathrm{CC}$ events in the detector occurring at $t \simeq \Delta / c$. For the beam parameters considered above, $70 \%$ of the events can be fully reconstructed observing the $\gamma$ pair in coincidence with the positron. For these events, the neutrino energy resolution $\sigma_{E_{v}}$ is $\sigma_{e m} \oplus \Delta_{p} / \sqrt{12}$, where $\sigma_{e m}$ is the e.m. energy resolution of the calorimeter and $\Delta_{p}$ is the momentum bite of the beamline. For the beam parameters of Sect. 3, this accuracy is dominated by $\Delta_{p}$ and $\sigma_{E_{v}}=(0.35 \oplus 0.49) \mathrm{GeV}=0.6 \mathrm{GeV}$ for $3 \mathrm{GeV}$ neutrinos. I.e. a momentum bite of $15 \%$ limits the relative precision of the neutrino energy reconstruction to $\sim 15 \%$.

An event by event tag facility must meet several challenges. An increase of the extraction time up to $T_{\text {extr }} \simeq 1 \mathrm{~s}$ makes the use of conventional horns unpractical. The focusing system will hence rely on static components, as discussed in Sect. 3. In addition, the momentum bite must be significantly smaller than the event count facility to fully exploit the $K_{e 3}$ kinematic reconstruction. Finally, due to the substantial increase in extraction time ( $1 \mathrm{~s}$ versus $2 \mathrm{~ms}$ ), the cosmic ray background in the neutrino detector is $\mathcal{O}(10 \times)$ the background in event count mode. Since a $v_{e}$ candidate must match a positron candidate at the calorimeter within $\delta$, the cosmic background contamination scales as $\mathcal{A} \cdot T_{2} / T_{1}$. Here, $T_{1}$ and $T_{2}$ are the extraction times in event count and event by event tag mode, respectively. For $T_{1}=2 \mathrm{~ms}, T_{2}=1 \mathrm{~s}$ and $\mathcal{A}=0.02$ the cosmic background increases by $\sim 10$.

In general, the event by event tag facility poses stronger technical challenges and, unlike the event count mode, its design will require a significant $R \& D$ phase.

\section{Conclusions}

Three body $K^{+} \rightarrow e^{+} \pi^{0} \nu_{e}$ decays in conventional neutrino beams offer unique opportunities to measure the $v_{e}$ charged current cross section with a precision of $\sim 1 \%$. In this paper, we discussed a facility that identifies positrons in the decay tunnel using calorimetric techniques to tag the production of $v_{e}$ at source. The positron rate at the instrumented decay tunnel removes the most important systematics related with the knowledge of the initial flux. An overall tagging efficiency of $59 \%$ is achievable in a specific beamline configuration that enhances the $v_{e} / v_{\mu}$ ratio to $\sim 2 \%$ and reduces the $v_{e}$ contribution due to DIF to $<0.1 \%$. Local rates and pile-up are well below the critical values for conventional 
calorimeters working at colliders. The integrated dose corresponding to $10^{4}$ events observed at the neutrino detector does not exceed $1.3 \mathrm{kGy}$. The construction of this facility, which monitors the positron production but does not associate uniquely the positron to the observed $v_{e}$ ("event count mode"), can be accomplished using existing technologies. For a 0.5 kton neutrino detector, the beam intensity needed to reach the $1 \%$ precision is well within reach of proton accelerators at CERN, Fermilab and JPARC.

The corresponding setup operated in event by event tag mode has been discussed, too. Event by event tagging, however, requires a purely static focusing system, a reduction on the secondary momentum bite and an overall time resolution $\delta$ of $\mathcal{O}(1)$ ns. Its implementation therefore implies additional $\mathrm{R} \& \mathrm{D}$ and advances in beam and detector technologies .

Acknowledgments The authors gratefully acknowledge discussions and suggestions from K. Anderson, A. Bross, N. Cartiglia, M. Dracos, J. Hylen, P. Loverre, M. Mezzetto, J. Morfin, B. Popov, C. Rubbia and T. Tabarelli.

Open Access This article is distributed under the terms of the Creative Commons Attribution 4.0 International License (http://creativecomm ons.org/licenses/by/4.0/), which permits unrestricted use, distribution, and reproduction in any medium, provided you give appropriate credit to the original author(s) and the source, provide a link to the Creative Commons license, and indicate if changes were made. Funded by $\mathrm{SCOAP}^{3}$.

\section{References}

1. J.A. Formaggio, G.P. Zeller, Rev. Mod. Phys. 84, 1307 (2012)

2. L. Alvarez-Ruso, Y. Hayato, J. Nieves, N. J. Phys. 16, 075015 (2014)

3. R. Gran et al., K2K Collaboration, Phys. Rev. D 74, 052002 (2006)

4. R. Gran et al., [K2K Collaboration], Nucl. Phys. Proc. Suppl. 221, $98(2011)$

5. P. Adamson et al. [MINOS Collaboration], Phys. Rev. D 81, 072002 (2010)

6. J. Dobson [T2K Collaboration], Nucl. Phys. Proc. Suppl. 199, $237-$ 238 (2013)

7. K. Abe et al. [T2K Collaboration], Phys. Rev. D 87, 092003 (2013)

8. K. Abe et al. [T2K Collaboration], Phys. Rev. D 90, 052010 (2014)

9. Y. Nakajima et al., [SciBooNE Collaboration], Phys. Rev. D 83, 012005 (2011)

10. B.G. Tice et al., [MINERvA Collaboration], Phys. Rev. Lett. 112, 231801 (2014)

11. R. Acciarri et al., [ArgoNeuT Collaboration], Phys. Rev. D 89, 112003 (2014)

12. For a review of latest results see F. Sanchez, Talk at XXVI International Conference on Neutrino Physics and Astrophysics, June 2-7 (Boston, 2014)

13. J. Blietschau et al. [Gargamelle Collaboration], Nucl. Phys. B 133, 205 (1978)

14. K. Abe et al. [T2K Collaboration], Phys. Rev. Lett. 113, 241803 (2014)

15. M. Day, K.S. McFarland, Phys. Rev. D 86, 053003 (2012)

16. S. Dusini, A. Longhin, M. Mezzetto, L. Patrizii, M. Sioli, G. Sirri, F. Terranova, Eur. Phys. J. C 73, 2392 (2013)
17. P. Coloma, P. Huber, J. Kopp, W. Winter, Phys. Rev. D 87, 033004 (2013)

18. C. Volpe, J. Phys. G 30, L1 (2004)

19. G.C. McLaughlin, Phys. Rev. C 70, 045804 (2004)

20. R.G.C. Oldeman, M. Meloni, B. Saitta, Eur. Phys. J. C 65, 81 (2010)

21. D. Adey et al., [nuSTORM Collaboration], arXiv:1308.6822 [physics.acc-ph]

22. J. Spitz, Phys. Rev. D 89, 073007 (2014)

23. L.N. Hand, A study of $40-90 \mathrm{GeV}$ neutrino interactions using a tagged neutrino beam. In: Proceedings of Second NAL Summer Study, Aspen, Colorado, 9 Jun-3 Aug 1969, p. 37

24. B. Pontecorvo, Lett. Nuovo Cim. 25, 257 (1979)

25. P. Denisov et al., IHEP, pp. 81-98, Serpukhov (1981, preprint)

26. R.H. Bernstein et al., FERMILAB-Proposal-0788 (1989)

27. L. Ludovici, P. Zucchelli [hep-ex/9701007]

28. L. Ludovici, F. Terranova, Eur. Phys. J. C 69, 331 (2010)

29. S. Geer, Phys. Rev. D 57, 6989 (1998) [Erratum-ibid. D 59 (1999) 039903]

30. P. Zucchelli, Phys. Lett. B 532, 166 (2002)

31. R. Baldy, J.L. Baldy, A.E. Ball, P. Bonnal, M. Buhler-Broglin, C. Detraz, K. Elsener, A. Ereditato et al., CERN-SL-99-034-DI, CERN-SL-99-34-DI, INFN-AE-99-05, INFN-AE-99-5

32. K.A. Olive et al. [Particle Data Group Collaboration], Chin. Phys. C 38, 090001 (2014)

33. E.H.M. Heijne, CERN-83-06, CERN-YELLOW-83-06

34. K. Anderson, J. Hylen, personal communication. See also K. Anderson, NuMI/NOvA 700kW Horn 1 Stripline Vibration Measurements. Talk at 9th International Workshop on Neutrino Beams and Instrumentation (NBI 2014)

35. T. Sekiguchi, T2K Horn Status. Talk at 9 th International Workshop on Neutrino Beams and Instrumentation (NBI 2014)

36. G. Battistoni, S. Muraro, P.R. Sala, F. Cerutti, A. Ferrari, S. Roesler, A. Fasso, J. Ranft, A.I.P. Conf. Proc. 896, 31 (2007)

37. A. Ferrari, P.R. Sala, A. Fasso, J. Ranft, CERN-2005-010, SLACR-773, INFN-TC-05-11. http://www.fluka.org

38. S. Ivanov, Accelerator Complex U70 of IHEP-Protvino: status and prospects for upgrade. In: Talk at 16th Lomonosov Conference on Elementary Particle Physics, August 22-28, Moscow, RU (2013)

39. K. Anderson et al., FERMILAB-DESIGN-1998-01 (1998)

40. A. Antonello et al., arXiv:1208.0862 [hep-ph]

41. S.K. Agarwalla et al., LAGUNA-LBNO Collaboration. JHEP 1405, 094 (2014)

42. S. Agostinelli et al. [GEANT4 Collaboration], Nucl. Instrum. Meth. A 506, 250 (2003)

43. J. Allison, K. Amako, J. Apostolakis, H. Araujo, P.A. Dubois, M. Asai, G. Barrand, R. Capra et al., IEEE Trans. Nucl. Sci. 53, 270 (2006)

44. C. Adloff et al., [CALICE Collaboration], JINST 5, P05004 (2010)

45. For a review see e.g. F. Cavallari, PoS (EPS-HEP 2013) 490 and references therein. http://pos.sissa.it/

46. F. Simon, C. Soldner, L. Weuste, JINST 8, P12001 (2013)

47. N. Cartiglia, M. Baselga, G. Dellacasa, S. Ely, V. Fadeyev, Z. Galloway, S. Garbolino, F. Marchetto et al., JINST 9, C02001 (2014)

48. G. Aad et al. [ATLAS Collaboration], ATLAS-CONF-2010-007, ATLAS-COM-CONF-2010-007

49. F. Hahn et al. [NA62 Collaboration], NA62 Technical Design Document, NA62-10-07 (2010)

50. T. Abe et al. [ISS Detector Working Group Collaboration], JINST 4, T05001 (2009)

51. M. Antonello et al. [ICARUS Collaboration], JINST 9, P08003 (2014)

52. C. Rubbia, arXiv:1408.6431 [physics.ins-det]

53. S.E. Kopp, Phys. Rept. 439, 101 (2007) 